\title{
Utilization of Cytokeratins 7 and 20 Does Not Differentiate between Barrett's Esophagus and Gastric Cardiac Intestinal Metaplasia
}

\author{
I. A. Mohammed, M.D., C. J. Streutker, M.D., M.Sc., F.R.C.P.C., R. H. Riddell, M.D., F.R.C.P.C. \\ Department of Pathology and Molecular Medicine (IAM, CJS, RHR), McMaster University, Hamilton, \\ Ontario, Canada; Department of Laboratory Medicine (CJS), St. Michael's Hospital, Toronto, Ontario, \\ Canada; and Department of Pathology and Laboratory Medicine (RHR), Mt. Sinai Hospital, Toronto, \\ Ontario, Canada
}

Long segment Barrett's esophagus (LSBE) is a recognized risk factor for the development of esophageal dysplasia and carcinoma. However, the risk of dysplasia arising within intestinal metaplasia below a normal-appearing $\mathrm{Z}$-line (i.e., in native cardiac mucosa) is unknown. Regular endoscopic surveillance is required in patients with LSBE and is frequently performed in short segment BE (SSBE), but the need for surveillance in cardiac intestinal metaplasia (CIM) is unknown. Unfortunately IM arising in SSBE and immediately below a normal Z-line can be indistinguishable histologically on H\&E stains. Previous reports suggest that the appearance of superficial CK20 immunohistochemical staining accompanied by intermediate and deep CK7 positivity is characteristic of $\mathrm{BE}$, whereas CIM specimens show superficial and deep CK20 positivity and weak to absent CK7 staining. We hypothesized that CK7/20 immunostaining of metaplastic biopsies from the esophagus and stomach would allow complete differentiation of these two entities when correlated with the endoscopic appearance. We undertook an evaluation of gastric and esophageal specimens to determine whether these characteristics were valid. Cases of both BE (long and short segment) and CIM, as well as cases of gastric cardiac biopsies lacking IM, were evaluated for CK7 and CK20 and correlated with the endoscopic appearance. We observed that, although the "Barrett's" pattern of $C K 7 / 20$ was maintained for many cases of BE, the sensitivity and specificity were only moderate (65\% and $56 \%$, respectively). The pattern

Copyright ( $\odot 2002$ by The United States and Canadian Academy of Pathology, Inc.

VOL. 15, NO. 6, P. 611, 2002 Printed in the U.S.A.

Date of acceptance: February 18, 2002.

Address reprint requests to: Dr. R. H. Riddell, Department of Pathology and Laboratory Medicine, Suite 600, Mt. Sinai Hospital, 600 University Avenue, Toronto, Ontario, Canada; e-mail: riddell@mtsinai.on.ca; fax: 416-586-8481. of staining for the CIM was variable, i.e., some cases showed a CK7/20 Barrett's pattern despite a normal appearance at endoscopy. The differences between this and previous studies may be due to inaccurate visualization of SSBE on endoscopy, the development of very early SSBE cases, inter-observer variability, fixation differences, or antibody differences. Whatever the cause of the differences, if results between laboratories are not comparable, CK7/20 immunostaining cannot be used to differentiate reliably between IM present in biopsy specimens taken from above versus below the Z-line. However, further studies should be performed to determine whether the presence or absence of a Barrett's pattern of CK7/20 immunostaining could predict progression to dysplasia or carcinoma.

KEY WORDS: Barrett's esophagus, Cardiac intestinal metaplasia, Cytokeratin.

Mod Pathol 2002;15(6):611-616

Barrett's esophagus (BE) is defined as the presence of specialized columnar epithelium with intestinal goblet cells, replacing the normal squamous epithelium (1-6). BE is generally subdivided into long segment BE (LSBE), where columnar mucosa extends $3 \mathrm{~cm}$ or more above the gastroesophageal junction (GEJ), and short segment BE (SSBE), where the specialized columnar epithelium is restricted to less than $3 \mathrm{~cm}$ above the GEJ. These definitions were, in the past, based on endoscopic evaluation. However, it has been recognized that histological identification of intestinal metaplasia is of particular importance, as it is this form of mucosa that is premalignant in nature and in which dysplasia and adenocarcinomas arise (7). The risk of progression from intestinal metaplasia to dysplasia and carcinoma is well recognized in LSBE, though it is now thought to be lower than previously estimated, at approximately $0.5 \%$ annually (8). Patients with 
LSBE are therefore regularly screened to evaluate any endoscopic or histological progression that would indicate the need for more aggressive therapy. The specific incidence of adenocarcinoma in SSBE is not well quantified, though Rudolph et al. (9) have shown that the risk for SSBE is essentially the same as for LSBE. Surveillance endoscopy in patients with SSBE, though still controversial, is frequently performed (10).

The gastric cardia is defined as the most proximal portion of the stomach, immediately distal to the normal GEJ (Z-line). Cardiac-type mucosa is defined as mucous glands that lack endocrine cells but may contain occasional parietal cells within the pits. It is thought to extend endoscopically distally for up to $2 \mathrm{~cm}$ from the GEJ in healthy individuals (11), although it is much shorter histologically. However, an autopsy study has noted that the presence and extent of cardiac mucosa increases with age (12). The finding of cardiac-type mucosa in the lower esophagus, which does not meet the definition of $\mathrm{BE}$, has been correlated with the presence and severity of gastroesophageal reflux disease (GERD) (13). These studies suggest that cardiactype mucosa may not be a normal finding in either the stomach or esophagus. Cardiac-type mucosa may itself be metaplastic and, therefore, may be a precursor to cardiac intestinal metaplasia (CIM). CIM is defined as the presence of intestinal metaplasia within cardiac-type mucosa below an endoscopically normal Z-line. The etiology of CIM may be different from $\mathrm{BE}$. The main risk factor for $\mathrm{BE}$ is GERD (13-15). Although the presence of Helicobacter pylori infection is linked to CIM $(16-20)$, in the absence of $H$. pylori infection CIM may be related to GERD (21). CIM may therefore have several etiologies. Similar to SSBE, the risk of development of dysplasia and adenocarcinoma in CIM is unknown. However, clinical studies suggest that the tendency for developing dysplasia or carcinoma in patients with intestinal metaplasia of the cardia is considerably lower than in $\mathrm{BE}(16,22)$. Consequently, patients with CIM are not usually enrolled in screening programs. Considering the marked rise in the incidence of gastric cardiac adenocarcinoma in conjunction with esophageal adenocarcinoma, this may change in the future $(23,24)$.

These differences in treatment protocols and risk of malignancy between LSBE, SSBE, and CIM indicate the need for accurate diagnosis of the different entities. However, differentiating these entities can be very difficult. Endoscopically, an irregular Z-line can be a normal finding, and determination of where the esophagus ends and the cardia begins can be unreliable. Furthermore, histological examination of biopsy specimens is frequently of little assistance because the appearance of intestinal metaplasia is identical in both locations. The pres- ence of squamous-lined ducts below the surface can help to identify the tissue as esophagus; however, these are rarely identified. Staining for acid mucins and specifically sulfomucins has been suggested as a means of identifying esophageal IM (25-27); however, this has not been found to be reliable $(19,28)$. Immunohistochemical staining of different cytokeratin subunits, particularly cytokeratins 7 and 20, has been proposed as a method that will differentiate them $(28,29)$.

In our study, we evaluated CK7 and CK20 pattern in patients with BE (both long and short segments), CIM, antral intestinal metaplasia (AIM), and normal/ inflamed cardiac biopsies to determine whether the staining pattern of these two antibodies would assist in diagnosis.

\section{MATERIALS AND METHODS}

\section{Case Selection}

Endoscopic biopsy specimens were identified by searching the information system for specific diagnostic categories. These diagnoses were based on standard H\&E staining. Diagnostic categories included esophageal mucosa with intestinal metaplasia (SSBE and LSBE), cardiac mucosa with intestinal metaplasia (CIM), antral mucosa with intestinal metaplasia (AIM), and normal/inflamed cardiac mucosa. In this last category, the cases of normal and inflamed cardia were combined as representatives of mūcosa lacking intestinal metaplasia. In all cases, the endoscopy report was consulted to determine the state of the Z-line, the presence or absence of an endoscopically-evident hiatus hernia, and to confirm the location of the biopsies. In cases where biopsies were labeled as "GEJ," these reports were used to determine whether there was any indication of SSBE. In total, 49 cases of BE, 13 cases of CIM, 7 cases of AIM, and 11 cases of nor$\mathrm{mal} /$ inflamed cardia were retrieved for the this study. In all cases, the biopsy specimens were formalin-fixed for approximately 24 hours. They then were processed and paraffin-embedded. Sections of 3- $\mu \mathrm{m}$ thickness were cut. H\&E-stained sections were examined. Alcian blue/periodic acidSchiff staining was utilized to confirm the presence of intestinal-type goblet cells.

\section{Immunohistochemistry}

Antibodies to CK7 and CK20 were utilized for this study as follows. The OV-TL clone (DAKO Diagnostics, Mississauga, Ontario, Canada) was utilized for CK7. For CK20, the Ks 20.8 clone was used. For both antibodies, slides were digested with Pronase followed by antibody at a dilution of 1:50 for $32 \mathrm{~min}$ - 
utes. Detection was performed with the Zymed goat anti-mouse streptavidin-biotin system.

Evaluation of the pattern of CK7/20 immunohistochemical staining was performed simultaneously by three pathologists (IM, CJS, RHR), two of whom were blinded to the diagnosis. The Barrett's CK7/20 pattern was considered present if CK20 staining was seen in surface epithelium with or without superficial and deep glands and CK7 staining was present in both superficial and deep glands in areas of IM. Statistical analysis of results was performed using the Fisher's exact test.

\section{RESULTS}

Review of patient history where available revealed that the majority of the patients with $\mathrm{BE}$ were male $(77 \%)$, which was comparable with the CIM patients (62\%) (Table 1). Few patients with BE had associated $H$. pylori infection (3 patients $=6 \%$ ), while most had a history of GERD and/or hiatus hernia $(69 \%$ and $35 \%)$. The presence of hiatus hernia may be underestimated due to our reliance on endoscopic reports for this diagnosis. Patients with CIM had similar GERD and hiatus hernia rates $(92 \%$ and $31 \%$ ), and three patients were diagnosed as having concurrent Helicobacter infection (25\%).

A total of 49 biopsies from patients clinically diagnosed with BE were evaluated for immunohistochemical staining pattern (Table 2). A Barrett's CK7/20 staining pattern was identified in 32 of the 48 slides (65\%) (Fig. 1). When subdivided into LSBE versus SSBE, 10 of 22 LSBE (54\%) and 22 of 27 SSBE $(81 \%)$ had a Barrett's pattern. The remainder of the cases had a variety of immunohistochemical staining patterns with the two antibodies, which did not conform to the staining pattern as previously established (Fig. 2).

In contrast, of the CIM biopsies, 9 of the 13 cases (69\%) showed a non-Barrett's CK7/20 pattern (Fig. 3 ), while the remaining four biopsies demonstrated the characteristic BE CK7/20 pattern (Fig. 4). A similar trend was noted in the biopsies of normal or inflamed cardiac mucosa-in these cases where histological evidence of intestinal metaplasia was lacking, 55\% had a Barrett's pattern of staining. Antral intestinal metaplasia also was capable of demonstrating a BE pattern of staining, though at a lower incidence (29\%).

In evaluating the utility of this test for diagnosis of $\mathrm{BE}$ versus CIM, the sensitivity was $65 \%$, specificity was $69 \%$, positive predictive value was $89 \%$, and negative predictive value was $35 \%$. By Fisher's exact test, there was no significant difference in the presence of non-BE patterns in CIM as opposed to BE patterns in all BE cases (2-tailed $P=.0547$ ). There was a significant difference when cases of CIM were compared only with SSBE (2-tailed $P=.0054$ ).

\section{DISCUSSION}

Cytokeratins (CK) are subunits of epithelial cell intermediate filaments, which are genetically determined. There is some restriction of the types of cytokeratins expressed in different epithelium. CK7 is a $54 \mathrm{kd}$ polypeptide found in normal epithelium ranging from lung, cervix, breast, and bile ducts to bladder transitional cell epithelium; however, it is essentially absent from gastrointestinal epithelia and squamous cell epithelium. CK20 expression is limited to intestinal epithelium and gastric foveolar cells as well as urothelial umbrella cells and Merkel cells of the epidermis $(30,31)$. Aberrant expression of cytokeratins may be observed in dysplastic and malignant cells. Previous studies attempted to differentiate between BE and CIM using antibodies to cytokeratins 7 and $20(28,29)$, though the utility of utilizing immunohistochemistry to differentiate these entities has been argued (32). Ormsby et al. demonstrated an apparently unique pattern of immunoreactivity, designated the BE CK7/20 pattern, showing superficial CK20 staining and strong CK7 staining of both superficial and deep glands in $100 \%$ of esophageal biopsy specimens from patients with $\operatorname{LSBE}(28,29)$. In contrast, a Barrett's CK7/20 pattern was not observed in those patients with gastric cardiac biopsies showing evidence of intestinal metaplasia. For LSBE, the sensitivity in this study was $97 \%$ with a specificity of $100 \%$. Our findings did not support this conclusion. Cases of both LSBE and SSBE showed marked variability in staining patterns, as did the biopsies from patients with CIM. This resulted in relatively poor sensitivity and specificity. There was a significant difference $(P$

TABLE 1. Clinical Characteristics of Patients with Barrett's Esophagus, Cardiac Intestinal Metaplasia, and Antral Intestinal Metaplasia

\begin{tabular}{lcccc}
\hline Clinical History & $\begin{array}{c}\text { Long Segment } \\
\text { Barrett's } \\
\text { Esophagus }\end{array}$ & $\begin{array}{c}\text { Short Segment } \\
\text { Barrett's } \\
\text { Esophagus }\end{array}$ & $\begin{array}{c}\text { Cardiac } \\
\text { Intestinal } \\
\text { Metaplasia }\end{array}$ & $\begin{array}{c}\text { Antral } \\
\text { Intestinal } \\
\text { Metaplasia }\end{array}$ \\
\hline Male gender $(n)$ & 13 & 23 & 12 & 3 \\
GERD $(n)$ & 9 & 24 & 12 & 2 \\
Hiatus hernia $(n)$ & 4 & 12 & 6 & 0 \\
H. pylori positive $(n)$ & 0 & 3 & 3 & 2 \\
\hline
\end{tabular}


TABLE 2. CK 7/20 Immunostaining Patterns

\begin{tabular}{ccccccc}
\hline Pattern & BE & LSBE & SSBE & CIM & AIM & Inflamed or Normal \\
& $(n=49)$ & $(n=22)$ & $(n=27)$ & $(n=13)$ & $(n=7)$ & 6 \\
Cardia $(n=11)$
\end{tabular}

BE, Barrett's esophagus; LSBE, long segment Barrett's esophagus; SSBE, short segment Barrett's esophagus; CIM, cardiac intestinal metaplasia; AIM, antral intestinal metaplasia.
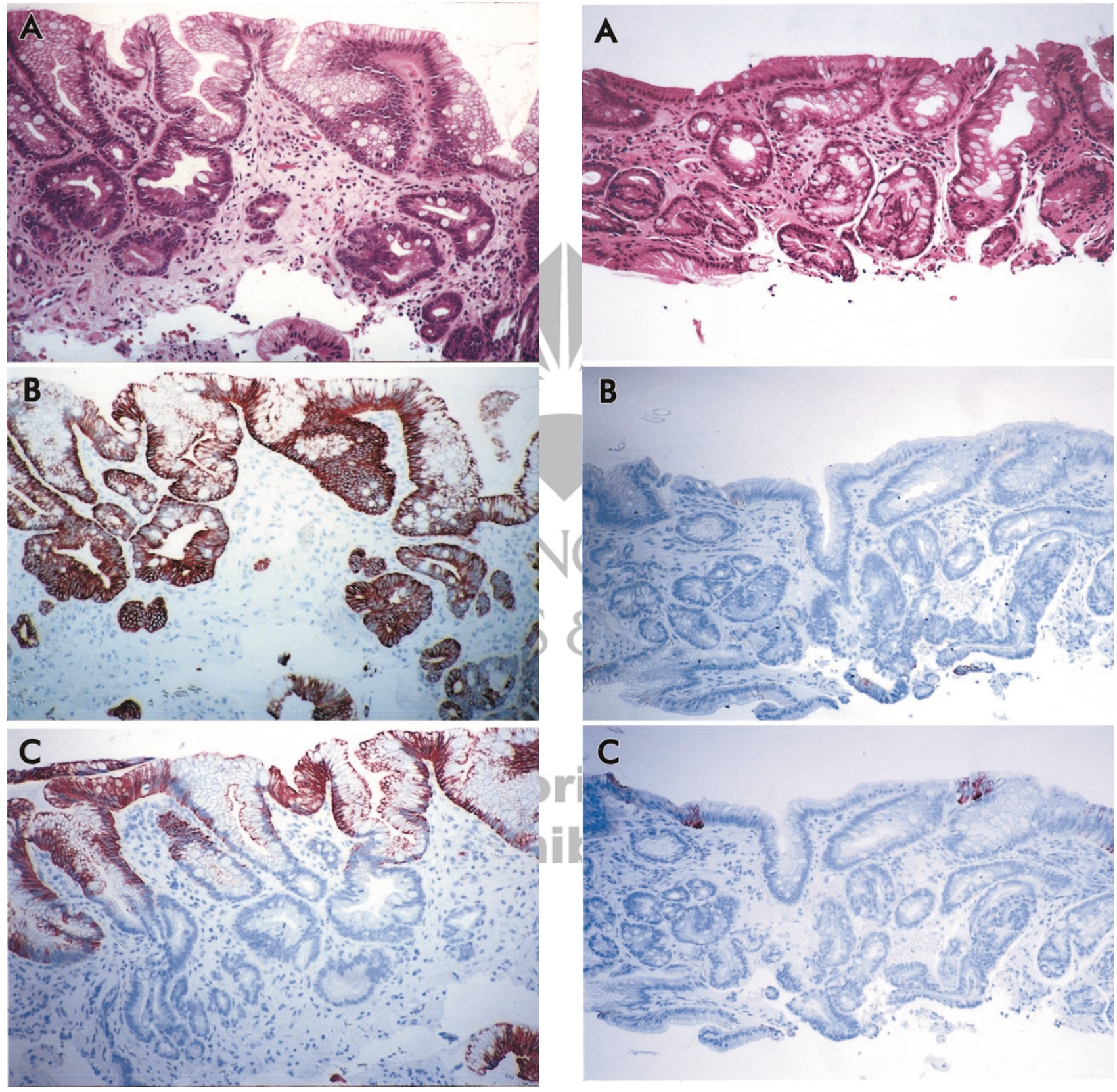

FIGURE 1. Barrett's esophagus with Barrett's CK7/20 pattern. (A) H\&E, (B) CK7, (C) CK20.

FIGURE 2. Barrett's esophagus with non-Barrett's CK7/20 pattern. (A) H\&E, (B) CK7, (C) CK20.

$=.0054)$ when the proportion of CIM with non-BE patterns was compared with SSBE (by Fisher's exact test).

The differences between this study and the previously reported results may have been due to a

less-strict interpretation of the specific Barrett's pattern of CK7/20 immunoreactivity compared with the original study. However, for this method of evaluation to be practical in the average surgical 

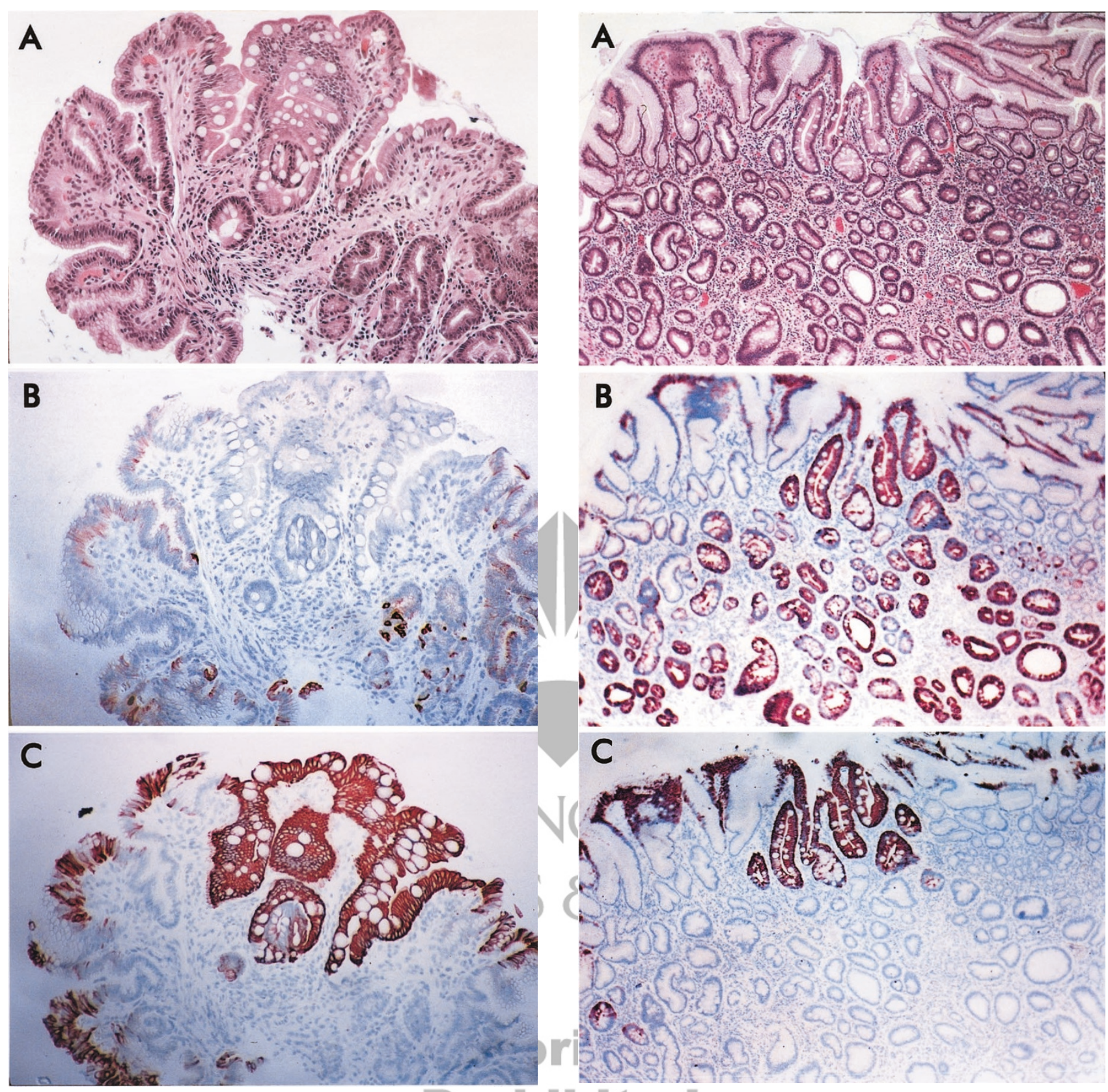

FIGURE 3. Cardiac intestinal metaplasia with non-Barrett's CK7/20 pattern. (A) H\&E, (B) CK7, (C) CK20.

pathology laboratory, it must be applicable despite reasonable inter-observer variability. The differences also may be due to inaccuracies in determining location at the time of endoscopy, or biopsies may have been taken from areas of very short SSBE. Differences in fixation methods or in the antibodies used for immunohistochemistry also could account for the variability in staining patterns. Regardless of the cause, the fact that these differences exist indicates that using cytokeratin profiles to distinguish $\mathrm{BE}$ from CIM is not likely to be clinically useful. Despite the presence of a significant difference between CIM and SSBE, in individual cases this

method still would not suffice to distinguish the two.

Though our results demonstrate that this methodology does not strictly divide cases of $\mathrm{BE}$ and CIM, they raise questions regarding the association of the BE pattern and subsequent risk of carcinoma. The previous studies note that in the cases where anomalous staining was found for either BE or CIM that these cases behaved according to the manner in which they stained, i.e., $\mathrm{BE}$ with a non-BE CK7/20 pattern acted like CIM. It may therefore be possible to link the pattern of CK7/20 immunohistochemical staining, irrespective of the endoscopic location of the biopsy, to the risk of development of 
carcinoma. However, this has not been extensively studied and would require a long-term prospective study.

\section{REFERENCES}

1. Paull A, Trier JS, Dalton MD, Camp RC, Loeb P, Goyal RK. The histologic spectrum of Barrett's esophagus. N Engl J Med 1976;295:476-80.

2. Reid BJ, Weinstein WM, Lewin KJ, Haggitt RC, VanDeventer G, DenBesten L, et al. Endoscopic biopsy can detect highgrade dysplasia or early adenocarcinoma in Barrett's esophagus without grossly recognizable neoplastic lesions. Gastroenterology 1988;94:81-90.

3. Spechler SJ, Robbins AH, Rubins HB, Vincent ME, Heeren T, Doos WG, et al. Adenocarcinoma and Barrett's esophagus. An overrated risk? Gastroenterology 1984;87:927-33.

4. Zwas F, Shields HM, Doos WG, Antonioli DA, Goldman H, Ransil BJ, et al. Scanning electron microscopy of Barrett's epithelium and its correlation with light microscopy and mucin stains. Gastroenterology 1986;90:1932-41.

5. Hamilton SR, Smith RR. The relationship between columnar epithelial dysplasia and invasive adenocarcinoma arising in Barrett's esophagus. Am J Clin Pathol 1987;87:301-12.

6. Schmidt HG, Riddell RH, Walther B, Skinner DB, Riemann JF. Dysplasia in Barrett's esophagus. J Cancer Res Clin Onco 1985;110:145-52.

7. DeMeester SR, Demeester TR. Columnar mucosa and intestinal metaplasia of the esophagus: fifty years of controversy. Ann Surg 2000;231:303-21.

8. Falk GW. Gastroesophageal reflux disease and Barrett's esophagus. Endoscopy 2001;33:109-18.

9. Rudolph RE, Vaughan TL, Storer BE, Haggitt RC, Rabinovitch PS, Levine DS, et al. Effect of segment length on risk for neoplastic progression in patients with Barrett esophagus. Ann Intern Med 2000;132:612-20.

10. Sharma P. Recent advances in Barrett's esophagus: shortsegment Barrett's esophagus and cardia intestinal metaplasia. Semin Gastrointest Dis 1999;10:93-102.

11. Hayward J. The lower end of the esophagus. Thorax 1961; $16: 36-41$

12. Chandrasoma PT, Der R, Ma Y, Dalton P, Taira M. Histology of the gastroesophageal junction: an autopsy study. Am J Surg Pathol 2000;24:402-09.

13. Chandrasoma PT, Lokuhetty DM, Demeester TR, Bremmer CG, Peters JH, Oberg S, et al. Definition of histopathologic changes in gastroesophageal reflux disease. Am J Surg Pathol 2000;24:344-51.

14. Carton E, Mulligan ED, Keeling PW, Tanner A, McDonald G, Reynolds JV. Specialized intestinal metaplasia: analysis of prevalence, risk factors and association with gastrooesophageal reflux disease. Br J Surg 2000;87:362-73.

15. El-Serag HB, Sonnenberg A, Jamal MM, Kunkel D, Crooks L, Feddersen RM. Characteristics of intestinal metaplasia in the gastric cardia. Am J Gastroenterol 1999;94:622-7.

16. Goldblum JR. Inflammation and intestinal metaplasia of the gastric cardia: Helicobacter pylori, gastroesophageal reflux disease, or both. Dig Dis 2000;18:14-9.

17. Hoshi T, Sasano H, Kato K, Ohara S, Shimosegawa T, Toyota $\mathrm{T}$, et al. Cell damage and proliferation in human gastric mucosa infected by Helicobacter pylori-a comparison before and after $\mathrm{H}$ pylori eradication in non-atrophic gastritis. Hum Pathol 1999;30:1412-7.

18. Jedrychowski W, Popiela T, Drews M, Gabryelewicz A, Marlicz K, Misiunia P, et al. Effect of Helicobacter pylori infection, smoking and dietary habits on the occurrence of antrum intestinal metaplasia. Clinico-epidemiological study in Poland. Pol J Pathol 1999;50:289-95.

19. Ormsby AH, Kilgore SP, Goldblum JR, Richter JE, Rice TW, Gramlich TL. The location and frequency of intestinal metaplasia at the esophagogastric junction in 223 consecutive autopsies: implications for patient treatment and preventive strategies in Barrett's esophagus. Mod Pathol 2000;13:61420.

20. Smith VC, Genta RM. Role of Helicobacter pylori gastritis in gastric atrophy, intestinal metaplasia, and gastric neoplasia. Microsc Res Tech 2000;48:313-20.

21. Goldstein NS, Karim R. Gastric cardia inflammation and intestinal metaplasia: association with reflux esophagitis and Helicobacter pylori. Mod Path 1999;12:1017-24.

22. Sharma P, Weston AP, Morales T, Topalovski M, Mayo MS, Sampliner RE. Relative risk of dysplasia for patients with intestinal metaplasia in the distal oesophagus and in the gastric cardia. Gut 2000;46:9-13.

23. Devesa SS, Fraumeni JF. The rising incidence of gastric cardia cancer. J Natl Cancer Inst 1999;91:747-9.

24. Sampliner RE. Adenocarcinoma of the esophagus and gastric cardia: is there progress in the face of increasing cancer incidence? Ann Intern Med 1999;130:67-9.

25. Byrne JP, Bhatnagar S, Hamid B, Armstrong GR, Attwood SE. Comparative study of intestinal metaplasia and mucin staining at the cardia and esophagogastric junction in 225 symptomatic patients presenting for diagnostic open-access gastroscopy. Am J Gastroenterol. 1999;94:98-103.

26. Chen YY, Wang HH, Antonioli DA, Spechler SJ, Zeroogian JM, Goyal R, et al. Significance of acid-mucin-positive nongoblet columnar cells in the distal esophagus and gastroesophageal junction. Hum Pathol 1999;30:1488-95.

27. Sirigu F, Capeccioni S, Dessi A. Mucin histochemistry in Barrett's esophagus. Riv Eur Sci Med Farmacol 1990;12:229 32.

28. Ormsby AH, Vaezi MF, Richter JE, Goldblum JR, Rice TW, Falk GW, et al. Cytokeratin immunoreactivity patterns in the diagnosis of short-segment Barrett's esophagus. Gastroenterology 2000;119:683-90.

29. Ormsby AH, Goldblum JR, Rice TW, Richter JE, Falk GW, Vaezi MF, et al. Cytokeratin subsets can reliably distinguish Barrett's esophagus from intestinal metaplasia of the stomach. Hum Pathol 1999;30:288-94.

30. Moll R, Lowe A, Laufer J, Franke WW. Cytokeratin 20 in human carcinomas. A new histodiagnostic marker detected by monoclonal antibodies. Am J Pathol 1992;140:427-47.

31. Ramaekers F, van Niekerk C, Poels L, Schaafsma E, Huijsmans A, Robben $\mathrm{H}$, et al. Use of monoclonal antibodies to keratin 7 in the differential diagnosis of adenocarcinomas. Am J Pathol 1990;136:641-55.

32. Glickman JN, Wang H, Das KM, Goyal RK, Spechler SJ, Antonioli D, et al. Phenotype of Barrett's esophagus and intestinal metaplasia of the distal esophagus and gastroesophageal junction: an immunohistochemical study of cytokeratins 7 and 20, Das-1 and 45 MI. Am J Surg Pathol 2001;25:87-94. 